\title{
Existence of Traveling Waves in Lattice Dynamical Systems
}

\author{
Xiaojun Li, Yong Jiang, Ziming Du \\ School of Science, Hohai University, Nanjing, China \\ Email: lixjun05@hhu.edu.cn, lixiaoj@yahoo.com
}

Received 28 December 2015; accepted 12 July 2016; published 15 July 2016

\begin{abstract}
Existence of traveling wave solutions for some lattice differential equations is investigated. We prove that there exists $c_{*}>0$ such that for each $c \geq c_{*}$, the systems under consideration admit monotonic nondecreasing traveling waves.
\end{abstract}

Keywords

Traveling Wave, Lattice Dynamical Systems, Schauder's Fixed Point Theorem

\section{Introduction}

Consider the following lattice differential equation

$$
\left\{\begin{array}{l}
\dot{u}_{i}=v\left(u_{i+1}-2 u_{i}+u_{i-1}\right)-f\left(u_{i},(B u)_{i}\right)+\alpha v_{i}, \quad i \in \mathrm{Z}, \\
\dot{v}_{i}=-\sigma v_{i}+\beta u_{i}, \quad i \in \mathrm{Z}
\end{array}\right.
$$

where $v, \sigma$ are positive constants, $\alpha \beta>0, f$ is a $C^{2}$-function, and $(B u)_{i}=u_{i+1}-u_{i}$.

Lattice dynamical systems occur in a wide variety of applications, and a lot of studies have been done, e.g., see [1]-[4]. A pair of solutions $\left\{u_{i}\right\}_{i=-\infty}^{\infty},\left\{v_{i}\right\}_{i=-\infty}^{\infty}$ of (1.1) is called a traveling wave solution with wave speed $c>0$ if there exist functions $U, V: R \rightarrow R$ such that $u_{i}=U(i+c t), v_{i}=V(i+c t)$ with $(U(-\infty), V(-\infty))=\left(U_{-}, V_{-}\right)$and $(U(+\infty), V(+\infty))=\left(U_{+}, V_{+}\right)$. Let $\xi=i+c t$, note that (1.1) has a pair of traveling wave solutions if and only if $U, V$ satisfy the functional differential equation

$$
\left\{\begin{array}{l}
c \dot{U}(\xi)=v(U(\xi+1)-2 U(\xi)+U(\xi-1))-f(U(\xi), B U(\xi))+\alpha V(\xi), \\
c \dot{V}(\xi)=-\sigma V(\xi)+\beta U(\xi) .
\end{array}\right.
$$

Without loss of generality, we can impose (1.1) with asymptotic boundary conditions

$$
\lim _{\xi \rightarrow-\infty} U(\xi)=0, \lim _{\xi \rightarrow+\infty} U(\xi)=k_{1}, \lim _{\xi \rightarrow-\infty} V(\xi)=0, \lim _{\xi \rightarrow+\infty} V(\xi)=k_{2} .
$$

By the property of equation, we can assume that $\alpha, \beta>0$. In the following, we give some assumptions on 
nonlinear function $f$ :

$$
\left(A_{1}\right)-f\left(k_{1}, 0\right)+\alpha k_{2}=0, \quad f(0,0)=0,-\sigma k_{2}+\beta k_{1}=0 .
$$

$\left(A_{2}\right)$ There exists a positive-value continuous function $Q: R \rightarrow R$ such that

$$
\begin{gathered}
\max _{u_{i},\left(B u_{i} \in[-r, r]\right.}\left|f_{u_{i}}^{\prime}\left(u_{i},(B u)_{i}\right)\right|+\max _{u_{i},\left(B u_{i} \in[-r, r]\right.}\left|f_{(B u)_{i}}^{\prime}\left(u_{i},(B u)_{i}\right)\right| \leq Q(r), Q\left(2 k_{1}\right)<v . \\
\left(A_{3}\right)-v<\frac{\partial f}{\partial x_{2}}(0,0)<0, \frac{\partial f}{\partial x_{1}}(0,0)<-3 v+\alpha \kappa-\frac{\beta}{\kappa}, \kappa=\frac{k_{2}}{k_{1}} . \\
\left(A_{4}\right) \frac{\partial^{2} f}{\partial x_{i} \partial x_{j}}\left(x_{1}, x_{2}\right)>0 \text { for any }\left(x_{1}, x_{2}\right) \in\left[0, k_{1}\right] \times[0, \omega], i, j=1,2,
\end{gathered}
$$

where $\omega=\left(e^{2 \Lambda_{*}}-1\right) k_{1}, \Lambda_{*}$ is given in Lemma 2.1.

$$
\left(A_{5}\right)-f(U(\xi), U(\xi+1)-U(\xi))+\alpha V(\xi) \neq 0 \text { for any }(U, V)(\xi) \in\left(0, k_{1}\right) \times\left(0, k_{2}\right) .
$$

Select positive constants $\mu_{1}, \mu_{2}$ such that $\mu_{1}>2 v+2 Q\left(2 k_{1}\right), \quad \mu_{2}>\sigma$, and define operators $H_{1}, H_{2}: C\left(R^{2}, R\right) \rightarrow C\left(R^{2}, R\right)$ by

$$
\begin{gathered}
H_{1}(U, V)(\xi)=\mu_{1} U(\xi)+v(U(\xi+1)-2 U(\xi)+U(\xi-1))-f(U(\xi), U(\xi+1)-U(\xi))+\alpha V(\xi) \\
H_{2}(U, V)(\xi)=\mu_{2} V(\xi)-\sigma V(\xi)+\beta U(\xi) .
\end{gathered}
$$

Then, (1.2) can be rewritten as

$$
c \dot{U}(\xi)=-\mu_{1} U(\xi)+H_{1}(U, V)(\xi), c \dot{V}(\xi)=-\mu_{2} V(\xi)+H_{2}(U, V)(\xi) .
$$

Define the operators $F_{i}: C\left(R^{2}, R\right) \rightarrow C\left(R^{2}, R\right)$ by

$$
F_{i}(U, V)(\xi)=\frac{1}{c} e^{-\frac{\mu_{i} \xi}{c}} \int_{-\infty}^{\xi} e^{\frac{\mu_{i} s}{c}} H_{i}(U, V)(s) d s, \quad i=1,2 .
$$

Note that $F_{i}$ satisfy $c F_{i}^{\prime}(U, V)(\xi)=-\mu_{i} F_{i}(U, V)(\xi)+H_{i}(U, V)(\xi), i=1,2$, and a fixed point of $F=\left(F_{1}, F_{2}\right)$ is a solution of (1.2). Denote $|\cdot|$ the Euclidean norm in $R^{2}$. Define

$$
B_{\mu}\left(R, R^{2}\right)=\left\{\Phi \in C\left(R, R^{2}\right): \sup _{t \in R}|\Phi(t)| e^{-\mu|t|}<\infty\right\},\|\Phi\|_{\mu}=\sup _{t \in R}|\Phi(t)| e^{-\mu|t|},
$$

where $0<\mu<\min \left\{\frac{\mu_{1}}{c}, \mu_{2}\right\}$. Note that $\left(B_{\mu}\left(R, R^{2}\right),\|\cdot\|_{\mu}\right)$ is a Banach space.

Definition 1.1. If the continuous functions $(\bar{U}(\xi), \bar{V}(\xi)): R \rightarrow R^{2}$ are differentiable almost everywhere and satisfy

$$
\left\{\begin{array}{l}
c \bar{U}^{\prime}(\xi) \geq v(\bar{U}(\xi+1)-2 \bar{U}(\xi)+\bar{U}(\xi-1))-f(\bar{U}(\xi), B \bar{U}(\xi))+\alpha \bar{V}(\xi), \\
c \bar{V}^{\prime}(\xi) \geq-\sigma \bar{V}(\xi)+\beta \bar{U}(\xi)
\end{array}\right.
$$

Then, $(\bar{U}(\xi), \bar{V}(\xi))$ is called an upper solution of (1.2).

Similarity, we can define a lower solution of (1.2). The main result of this paper is

Theorem 1.1. Assume that $\left(A_{1}\right)-\left(A_{5}\right)$ hold. Then there exists $c_{*}>0$ such that for every $c \geq c_{*},(1.2)$ admits a traveling wave solution $(U(\xi), V(\xi))$ connecting $(0,0)$ and $\left(k_{1}, k_{2}\right)$. Moreover, each component of traveling wave solution is monotonically nondecreasing in $\xi \in R$, and for each $c \geq c_{*}, U(\xi), V(\xi)$ also satisfy $\lim _{\xi \rightarrow-\infty} U(\xi) e^{-\Lambda_{1}(c) \xi}=1, \quad 0<\lim _{\xi \rightarrow-\infty} V(\xi) e^{-\Lambda_{1}(c) \xi} \leq \kappa$, where $\lambda=\Lambda_{1}(c)$ is the smallest solution of the equation

$$
c \lambda-\left[\left(v-\frac{\partial f}{\partial x_{2}}(0,0)\right) e^{\lambda}+v e^{-\lambda}\right]+2 v+\frac{\partial f}{\partial x_{1}}(0,0)-\frac{\partial f}{\partial x_{2}}(0,0)-\alpha \kappa=0 .
$$




\section{Upper-Lower Solutions of (1.2)}

Set $\Delta(c, \lambda)=c \lambda-\left[\left(v-\frac{\partial f}{\partial x_{2}}(0,0)\right) e^{\lambda}+v e^{-\lambda}\right]+2 v+\frac{\partial f}{\partial x_{1}}(0,0)-\frac{\partial f}{\partial x_{2}}(0,0)-\alpha \kappa$.

Lemma 2.1. Assume that $\left(A_{3}\right)$ holds. Then there exists a unique $c_{*}>0$ such that $(i)$ if $c>c_{*}$, then there exist two positive numbers $\Lambda_{1}(c)$ and $\Lambda_{2}(c)$ with $\Lambda_{1}(c)<\Lambda_{2}(c)$ such that $\Delta\left(c, \Lambda_{1}(c)\right)=\Delta\left(c, \Lambda_{2}(c)\right)=0, \Delta(c, \cdot)>0$ in $\left(\Lambda_{1}(c), \Lambda_{2}(c)\right)$, and $\Delta(c, \cdot)<0$ in $R \backslash\left[\Lambda_{1}(c), \Lambda_{2}(c)\right]$; (ii) if $c<c_{*}$, then $\Delta(c, \lambda)<0$ for all $\lambda \geq 0$; (iii) if $c=c_{*}$, then $\Lambda_{1}(c)=\Lambda_{2}(c)=\Lambda_{*}$, and $\Delta\left(c_{*}, \Lambda_{*}\right)=0$.

Proof. Using assumption $\left(A_{3}\right)$, we can get the result directly. $\square$

Lemma 2.2. Assume that $\left(A_{1}\right),\left(A_{3}\right)$ and $\left(A_{4}\right)$ hold. Let $c_{*}, \Lambda_{1}(c)$, and $\Lambda_{2}(c)$ be defined as in Lemma 2.1, and $c>c_{*}$ be any number. Then for every $\theta \in\left(1, \min \left\{\frac{\Lambda_{2}(c)}{\Lambda_{1}(c)}\right.\right.$, 2\}) and $0<h<\kappa$, there exists $Q(c, \theta) \geq 1$ such that for any $q \geq Q(c, \theta)$,

$$
\phi^{+}(\xi):=\min \left\{k_{1}, e^{\Lambda_{1}(c) \xi}+q e^{\theta \Lambda_{1}(c) \xi}\right\}, \psi^{+}(\xi):=\min \left\{k_{2}, \kappa\left(e^{\Lambda_{1}(c) \xi}+q e^{\theta \Lambda_{1}(c) \xi}\right)\right\}, \xi \in R
$$

and

$$
\phi_{-}(\xi):=\max \left\{0, e^{\Lambda_{1}(c) \xi}-q e^{\theta \Lambda_{1}(c) \xi}\right\}, \psi_{-}(\xi):=\max \left\{0, h\left(e^{\Lambda_{1}(c) \xi}-q e^{\theta \Lambda_{1}(c) \xi}\right)\right\}, \xi \in R
$$

are a pair of upper solutions and a pair of lower solutions of (1.2), respectively.

Proof. Let

$$
\begin{gathered}
N_{1}^{c}[\phi, \psi](\xi):=c \phi^{\prime}(\xi)-v[\phi(\xi+1)-2 \phi(\xi)+\phi(\xi-1)]+f(\phi(\xi), \phi(\xi+1)-\phi(\xi))-\alpha \psi(\xi), \\
N_{2}^{c}[\phi, \psi](\xi):=c \psi^{\prime}(\xi)+\sigma \psi(\xi)-\beta \psi(\xi) .
\end{gathered}
$$

Since $\kappa=\frac{k_{2}}{k_{1}}$, there exists $\xi_{1}$ such that $\phi^{+}\left(\xi_{1}\right)=k_{1}, \psi^{+}\left(\xi_{1}\right)=k_{2}$. If $\xi \geq \xi_{1}$, then $\phi^{+}(\xi)=k_{1}$, $\psi^{+}(\xi)=k_{2}$. By $\left(A_{1}\right)$, we get that

$$
N_{1}^{c}\left[\phi^{+}, \psi^{+}\right](\xi) \geq f\left(k_{1}, 0\right)-\alpha k_{2}=0, \quad N_{2}^{c}\left[\phi^{+}, \psi^{+}\right](\xi) \geq \sigma k_{2}-\beta k_{1}=0 .
$$

If $\xi<\xi$, then $\phi^{+}(\xi)=e^{\Lambda_{1}(c) \xi}+q e^{\theta \Lambda_{1}(c) \xi}, \psi^{+}(\xi)=\kappa\left(e^{\Lambda_{1}(c) \xi}+q e^{\theta \Lambda_{1}(c) \xi}\right)$. By $\left(A_{1}\right),\left(A_{3}\right)-\left(A_{4}\right)$, and using Lemma 2.1, we get that

$$
\begin{gathered}
N_{1}^{c}\left[\phi^{+}, \psi^{+}\right](\xi) \geq c\left(\Lambda_{1}(c) e^{\Lambda_{1}(c) \xi}+q \theta \Lambda_{1}(c) e^{\theta \Lambda_{1}(c) \xi}\right)-v\left[e^{\Lambda_{1}(c)(\xi+1)}+q e^{\theta \Lambda_{1}(c)(\xi+1)}-2 e^{\Lambda_{1}(c) \xi}\right. \\
\left.-2 q e^{\theta \Lambda_{1}(c) \xi}+e^{\Lambda_{1}(c)(\xi-1)}+q e^{\theta \Lambda_{1}(c)(\xi-1)}\right]+f\left(e^{\Lambda_{1}(c) \xi}+q e^{\theta \Lambda_{1}(c) \xi},\right. \\
\left.e^{\Lambda_{1}(c)(\xi+1)}+q e^{\theta \Lambda_{1}(c)(\xi+1)}-e^{\Lambda_{1}(c) \xi}-q e^{\theta \Lambda_{1}(c) \xi}\right)-\alpha \kappa\left(e^{\Lambda_{1}(c) \xi}+q e^{\theta \Lambda_{1}(\xi)}\right) \\
\geq \Delta\left(c, \Lambda_{1}(c)\right) e^{\Lambda_{1}(c) \xi}+\Delta\left(c, \theta \Lambda_{1}(c)\right) q e^{\theta \Lambda_{1}(c) \xi} \geq 0 .
\end{gathered}
$$

Lemma 2.1 and $\left(A_{3}\right)$ yields

$$
c \kappa \Lambda_{1}(c) \theta+\kappa \sigma-\beta>c \kappa \Lambda_{1}(c)+\kappa \sigma-\beta>0 .
$$

Thus,

$$
\begin{aligned}
N_{2}^{c}\left[\phi^{+}, \psi^{+}\right](\xi)= & c \kappa\left(\Lambda_{1}(c) e^{\Lambda_{1}(c) \xi}+q \theta \Lambda_{1}(c) e^{\theta \Lambda_{1}(c) \xi}\right) \\
& +\kappa \sigma\left(e^{\Lambda_{1}(c) \xi}+q e^{\theta \Lambda_{1}(c) \xi}\right)-\beta\left(e^{\Lambda_{1}(c) \xi}+q e^{\theta \Lambda_{1}(c) \xi}\right) \\
= & \left(c \kappa \Lambda_{1}(c)+\kappa \sigma-\beta\right) e^{\Lambda_{1}(c) \xi}+q\left(c \kappa \Lambda_{1}(c) \theta+\kappa \sigma-\beta\right) e^{\theta \Lambda_{1}(c) \xi}>0 .
\end{aligned}
$$

Therefore, $\left(\phi^{+}, \psi^{+}\right)(\xi)$ is an upper solution of (1.2). Similarly, we can prove that $\left(\phi_{-}, \psi_{-}\right)(\xi)$ is a lower 
solution. $\square$

\section{Existence of Traveling Wave}

Let $K=\left(k_{1}, k_{2}\right), C_{[0, K]}\left(R, R^{2}\right)=\left\{(U, V) \in C\left(R, R^{2}\right): 0 \leq U(s) \leq k_{1}, 0 \leq V(s) \leq k_{2}, s \in R\right.$. $\}$. We have the following result.

Lemma 3.1 Assume that $\left(A_{1}\right)$ and $\left(A_{2}\right)$ hold. Then

(i) $F_{1}\left(U_{1}, V_{1}\right)(\xi) \geq F_{1}\left(U_{2}, V_{2}\right)(\xi)$ and $F_{2}\left(U_{1}, V_{1}\right)(\xi) \geq F_{2}\left(U_{2}, V_{2}\right)(\xi)$ for $\xi \in R$ if $\left(U_{1}, V_{1}\right)(\xi),\left(U_{2}, V_{2}\right)(\xi) \in C_{[0, K]}\left(R, R^{2}\right)$ satisfy $U_{1}(\xi) \geq U_{2}(\xi), V_{1}(\xi) \geq V_{2}(\xi)$ for $\xi \in R$;

(ii) $F_{1}(U, V)(\xi), F_{2}(U, V)(\xi)$ are nondecreasing in $\xi \in R$ if $(U, V)(\xi) \in C_{[0, K]}\left(R, R^{2}\right)$ is nondecreasing in $\xi \in R$.

Proof. If $\left(U_{1}, V_{1}\right)(\xi),\left(U_{2}, V_{2}\right)(\xi) \in C_{[0, K]}\left(R, R^{2}\right)$ such that $U_{1}(\xi) \geq U_{2}(\xi)$ and $V_{1}(\xi) \geq V_{2}(\xi)$ for $\xi \in R$, then by $\left(A_{2}\right)$ we have

$$
\begin{aligned}
& \left|f\left(U_{1}(\xi), B U_{1}(\xi)\right)-f\left(U_{2}(\xi), B U_{2}(\xi)\right)\right| \\
& =\mid \int_{0}^{1}\left[f_{U}^{\prime}\left(U_{2}+\theta\left(U_{1}-U_{2}\right), B U_{2}+\theta\left(B U_{1}-B U_{2}\right)\right)\left(U_{1}-U_{2}\right)\right. \\
& \left.\quad+f_{B U}^{\prime}\left(U_{2}+\theta\left(U_{1}-U_{2}\right), B U_{2}+\theta\left(B U_{1}-B U_{2}\right)\right)\left(B U_{1}-B U_{2}\right)\right] d \theta \mid \\
& \leq 2 M_{1}\left(U_{1}(\xi)-U_{2}(\xi)\right)+M_{1}\left(U_{1}(\xi+1)-U_{2}(\xi+1)\right),
\end{aligned}
$$

where $M_{1}=Q\left(2 k_{1}\right)$. Note that

$$
\begin{aligned}
& H_{1}\left(U_{1}, V_{1}\right)(\xi)-H_{1}\left(U_{2}, V_{2}\right)(\xi) \\
& =\left(\mu_{1}-2 v\right)\left(U_{1}(\xi)-U_{2}(\xi)\right)+v\left[\left(U_{1}(\xi+1)-U_{2}(\xi+1)\right)+\left(U_{1}(\xi-1)-U_{2}(\xi-1)\right)\right] \\
& -\left[f\left(U_{1}(\xi), B U_{1}(\xi)\right)-f\left(U_{2}(\xi), B U_{2}(\xi)\right)\right]+\alpha\left(V_{1}(\xi)-V_{2}(\xi)\right) .
\end{aligned}
$$

Thus, from (3.1)-(3.2), we have

$$
\begin{aligned}
& H_{1}\left(U_{1}, V_{1}\right)(\xi)-H_{1}\left(U_{2}, V_{2}\right)(\xi) \\
& \geq\left(\mu_{1}-2 v-2 M_{1}\right)\left(U_{1}(\xi)-U_{2}(\xi)\right)+\left(v-M_{1}\right)\left(U_{1}(\xi+1)-U_{2}(\xi+1)\right) \\
& \quad+v\left(U_{1}(\xi-1)-U_{2}(\xi-1)\right)+\alpha\left(V_{1}(\xi)-V_{2}(\xi)\right) \geq 0,
\end{aligned}
$$

which implies that $H_{1}\left(U_{1}, V_{1}\right)(\xi) \geq H_{1}\left(U_{2}, V_{2}\right)(\xi)$. A similar argument can be done for $H_{2}(U, V)(\xi)$. Thus, we can get the desired results.

Lemma 3.2. Assume that $\left(A_{1}\right)$ and $\left(A_{2}\right)$ hold. Then $F=\left(F_{1}, F_{2}\right): B_{\mu}\left(R, R^{2}\right) \rightarrow B_{\mu}\left(R, R^{2}\right)$ is continuous with respect to the norm $\|\cdot\|_{\mu}$ with $0<\mu<\min \left\{\frac{\mu_{1}}{c}, \mu_{2}\right\}$.

Proof. We first prove that $H_{1}, H_{2}: B_{\mu}\left(R, R^{2}\right) \rightarrow B_{\mu}\left(R, R^{2}\right)$ are continuous. Denote $\Phi_{1}=\left(U_{1}, V_{1}\right)$, $\Phi_{2}=\left(U_{2}, V_{2}\right)$. For any $\varepsilon>0$, choose $0<\delta<\frac{\varepsilon}{N}$, where

$N=\max \left\{\mu_{1}-2 v+2 M_{1}+\left(2 v+M_{1}\right) e^{\mu}+\alpha, \mu_{2}-\sigma+\beta\right\}$. If $\Phi_{1}$ and $\Phi_{2}$ satisfy

$\left\|\Phi_{1}-\Phi_{2}\right\|_{\mu}=\sup _{\xi \in R}\left|\Phi_{1}(\xi)-\Phi_{2}(\xi)\right| e^{-\mu|\xi|}<\delta$, then by (3.1),

$$
\begin{aligned}
\mid & H_{1}\left(U_{1}, V_{1}\right)(\xi)-H_{1}\left(U_{2}, V_{2}\right)(\xi) \mid e^{-\mu|\xi|} \\
= & \mid\left(\mu_{1}-2 v\right)\left(U_{1}(\xi)-U_{2}(\xi)\right)+v\left[\left(U_{1}(\xi+1)-U_{2}(\xi+1)\right)+\left(U_{1}(\xi-1)-U_{2}(\xi-1)\right)\right] \\
& -\left(f\left(U_{1}(\xi), B U_{1}(\xi)\right)-f\left(U_{2}(\xi), B U_{2}(\xi)\right)\right)+\alpha\left(V_{1}(\xi)-V_{2}(\xi)\right) \mid e^{-\mu|\xi|} \\
\leq & {\left[\mu_{1}-2 v+2 M_{1}+\left(2 v+M_{1}\right) e^{\mu}+\alpha\right]\left\|\Phi_{1}(\xi)-\Phi_{2}(\xi)\right\|_{\mu}<\varepsilon . }
\end{aligned}
$$


Similarly, $H_{2}\left(U_{1}, V_{1}\right)(\xi)$ is continuous.

By definition of $F_{1}$, we have

$$
\begin{aligned}
& \left|F_{1}\left(U_{1}, V_{1}\right)(\xi)-F_{1}\left(U_{2}, V_{2}\right)(\xi)\right|=\frac{1}{c} e^{-\frac{\mu_{1}}{c} \xi}\left|\int_{-\infty}^{\xi}\left(H_{1}\left(U_{1}, V_{1}\right)-H_{1}\left(U_{2}, V_{2}\right)\right)(s) d s\right| \\
& \leq \frac{1}{c}\left\|H_{1}\left(U_{1}, V_{1}\right)(\xi)-H_{1}\left(U_{2}, V_{2}\right)(\xi)\right\|_{\mu} e^{-\frac{\mu_{1}}{c} \xi} \int_{-\infty}^{\xi} e^{\frac{\mu_{1}}{c} s+\mu|s|} d s .
\end{aligned}
$$

If $\xi<0$, it follows that

$$
\left|F_{1}\left(U_{1}, V_{1}\right)(\xi)-F_{1}\left(U_{2}, V_{2}\right)(\xi)\right| e^{-\mu|\xi|} \leq \frac{1}{\mu_{1}-c \mu}\left\|H_{1}\left(U_{1}, V_{1}\right)(\xi)-H_{1}\left(U_{2}, V_{2}\right)(\xi)\right\|_{\mu} .
$$

If $\xi \geq 0$, it follows that

$$
\begin{aligned}
& \left|F_{1}\left(U_{1}, V_{1}\right)(\xi)-F_{1}\left(U_{2}, V_{2}\right)(\xi)\right| e^{-\mu|\xi|} \\
& \leq\left[\left(\frac{1}{\mu_{1}-c \mu}-\frac{1}{\mu_{1}+c \mu}\right) e^{-\frac{\mu_{1}+c \mu}{c} \xi}+\frac{1}{\mu_{1}+c \mu}\right]\left\|H_{1}\left(U_{1}, V_{1}\right)(\xi)-H_{1}\left(U_{2}, V_{2}\right)(\xi)\right\|_{\mu} \\
& \leq \frac{1}{\mu_{1}-c \mu}\left\|H_{1}\left(U_{1}, V_{1}\right)(\xi)-H_{1}\left(U_{2}, V_{2}\right)(\xi)\right\|_{\mu} .
\end{aligned}
$$

Combining (3.5) and (3.6), we get that $F_{1}$ is continuous with respect to the norm $\|\cdot\|_{\mu}$. A Similar argument can be done for $F_{2}$.

Define

$$
\begin{aligned}
& \Gamma=\Gamma\left(\left[\phi_{-}, \psi_{-}\right],\left[\phi^{+}, \psi^{+}\right]\right) \\
& :=\left\{(\phi, \psi) \in C\left(R, R^{2}\right) \mid \begin{array}{c}
\text { (i) } \phi(\xi), \psi(\xi) \text { are nondecrea sin } g \text { in } R ; \\
\text { ii } \phi_{-}(\xi) \leq \phi(\xi) \leq \phi^{+}(\xi) \text { and } \psi_{-}(\xi) \leq \psi(\xi) \leq \psi^{+}(\xi) \\
\text { for all } \xi \in R ; \\
\text { iii }\left|\phi\left(\xi_{1}\right)-\phi\left(\xi_{2}\right)\right| \leq \frac{2 \mu_{1} k_{1}}{c}\left|\xi_{1}-\xi_{2}\right| \text { and } \mid \psi\left(\xi_{1}\right)- \\
\psi\left(\xi_{2}\right)\left|\leq \frac{2 \mu_{2} k_{2}}{c}\right| \xi_{1}-\xi_{2} \mid \text { for all } \xi_{1}, \xi_{2} \in R .
\end{array}\right\} .
\end{aligned}
$$

It is easy to verify that $\Gamma$ is nonempty, convex and compact in $B_{\mu}\left(R, R^{2}\right)$. As the proof of Claim 2 in the proof of Theorem A in [5], we have

Lemma 3.3. Assume that $\left(A_{1}\right)-\left(A_{3}\right)$ hold. Then $F(\Gamma) \subset \Gamma$.

Proof of Theorem 1.1. By the definition of $\Gamma$, Lemma 3.2-3.3 and Schauder's fixed point theorem, we get that there exists a fixed point $\left(\phi^{*}(\xi), \psi^{*}(\xi)\right) \in \Gamma$. Note that $\left(\phi^{*}(\xi), \psi^{*}(\xi)\right)$ is nondecreasing in $\xi \in R$, assumption $\left(A_{5}\right)$ and Lemma 2.2 imply that $\lim _{\xi \rightarrow-\infty}\left(\phi^{*}(\xi), \psi^{*}(\xi)\right)=(0,0), \lim _{\xi \rightarrow+\infty}\left(\phi^{*}(\xi), \psi^{*}(\xi)\right)=\left(k_{1}, k_{2}\right)$. Therefore, $\left(\phi^{*}(\xi), \psi^{*}(\xi)\right)$ is a traveling wave solution of (1.1).

\section{Acknowledgements}

This work was supported by the NNSF of China Grant 11571092.

\section{References}

[1] Chow, S., Mallet-Paret, J. and Shen, W. (1998) Traveling Waves in Lattice Dynamical Systems. Journal of Differential Equations, 149, 248-291. http://dx.doi.org/10.1006/jdeq.1998.3478

[2] Li, X. and Wang, D. (2007) Attractors for Partly Dissipative Lattice Dynamic Systems in Weighted Spaces. Journal of Mathematical Analysis and Applications, 325, 141-156. http://dx.doi.org/10.1016/j.jmaa.2006.01.054 
[3] Ma, S. and Zou, X. (2005) Existence, Uniqueness and Stability of Traveling Waves in Adiscrete Reaction-Diffusion Monostable Equation with Delay. Journal of Differential Equations, 217, 54-87. http://dx.doi.org/10.1016/j.jde.2005.05.004

[4] Zinner, B. (1992) Existence of Traveling Wavefront Solutions for the Discrete Nagumo Equation. Journal of Differential Equations, 96, 1-27. http://dx.doi.org/10.1016/0022-0396(92)90142-A

[5] Li, X. (2011) Existence of Traveling Wavefronts of Nonlocal Delayed Lattice Differential Equations. Journal of Dynamical and Control Systems, 17, 427-449. http://dx.doi.org/10.1007/s10883-011-9124-1

\section{Submit or recommend next manuscript to SCIRP and we will provide best service for you:}

Accepting pre-submission inquiries through Email, Facebook, LinkedIn, Twitter, etc.

A wide selection of journals (inclusive of 9 subjects, more than 200 journals)

Providing 24-hour high-quality service

User-friendly online submission system

Fair and swift peer-review system

Efficient typesetting and proofreading procedure

Display of the result of downloads and visits, as well as the number of cited articles

Maximum dissemination of your research work

Submit your manuscript at: http://papersubmission.scirp.org/ 